\title{
Implications of Environmental Stress during Seed Development on Reproductive and Seed Bank Persistence Traits in Wild Oat (Avena fatua L.)
}

Robert S. Gallagher ${ }^{1, *}$, Kristen L. Granger ${ }^{2}$, Amanda M. Snyder ${ }^{3}$, Dennis Pittmann ${ }^{3}$ and E. Patrick Fuerst ${ }^{3}$

1 W.P. Jacobs Institute for Sustainable Food Systems, 302 South Broad Street, Clinton, SC 29325, USA

2 Department of Crop and Soil Sciences, The Pennsylvania State University, University Park, PA 16801, USA; E-Mail: kgranger1@gmail.com

3 Department of Crop and Soil Sciences, Washington State University, Pullman, WA 99164, USA; E-Mails: amsnyder@uidaho.edu (A.M.S.); pittmann@wsu.edu (D.P.); pfuerst@wsu.edu (E.P.F.)

* Author to whom correspondence should be addressed; E-Mail: rsgallagher@gmail.com; Tel.: +1-864-547-1500.

Received: 2 July 2013; in revised form: 19 July 2013 / Accepted: 24 July 2013 /

Published: 30 July 2013

\begin{abstract}
Weeds produce seed under a wide range of conditions, depending on timing of emergence, prevailing crop, soil microsites, and climatic conditions, among other factors. We hypothesized that the maturation environment during weed seed development will influence reproductive allocation and seed persistence traits, such as seed dormancy and vigor, and needs to be considered when formulating weed management strategies. This research evaluated the effects of shade and drought stress on reproductive allocation, seed dormancy and seed vigor in select lines of wild oat (Avena fatua L.). Plants were grown in the greenhouse under drought stress and shade. Harvested seed were subjected to controlled after-ripening and aging regimes. Drought and shade reduced reproductive allocation and resulted in seed with less intense primary dormancy compared to the plants grown under resource-rich conditions, but had no apparent effect on seed vigor. Our data provide additional support to the hypothesis that seed dormancy within a species is a highly plastic trait that can be strongly influenced by the growth conditions of the mother plant. Such plasticity may have important implications for establishing ecologically-based weed control criteria on which threshold-based weed management systems are implemented.
\end{abstract}


Keywords: Avena fatua; maturation environment; seed bank; seed dormancy; seed vigor; reproductive allocation

\section{Introduction}

An important component of integrated pest management (IPM) is pest thresholds, which facilitates more judicious pesticide use by only applying pesticides when the potential damage from the pest exceeds the cost of the pesticide application. This approach has been considered for the management of crop weeds as means to reduce the reliance on herbicides, where overuse can lead to the contamination of ground and surface waters [1] and to the development of herbicide resistant weed populations [2]. Although sub-threshold weed communities may not be economically competitive with crops, they are still likely to produce seed, which may contribute to the long-term soil weed seed bank. Such contributions to the soil weed bank may be a major problem with threshold-based weed management, potentially leading to the proliferation of weed communities in the longer term [3].

Weeds, however, emerge and develop under a wide range of environmental conditions depending on time of emergence relative to the crop, the plant architecture of the crop, and crop management, among other factors. Sub-threshold weed communities in particular would be subject to considerable competition from the crop, potentially limiting soil resources (i.e., water and nutrients) as well as light. Such resource limitations will not only reduce reproductive output, but may also influence the seed traits associated with persistence in the soil seed bank (reviewed in [4]). For example, drought stress imposed at anthesis in wild oat (Avena fatua L.) reduced the intensity of primary physiological seed dormancy [5], and resulted in wild oat seed that emerged sooner and had a shorter seed bank persistence than seed from non-stressed plants [6]. We previously reported that light limitation or a cyclic drought regime resulted in reduced allocation to the palea/lemma as well as phenolic acid concentration of wild oat seed, which may serve as physical and chemical defense mechanisms, respectively [7].

The objective of this study was to evaluate the effects of the drought and shade regimes imposed on wild oat plants during seed development and seed maturation timing on reproductive output and allocation, primary physiological seed dormancy and seed/seedling vigor. Reproductive output is an indicator of the plants' ability to contribute to the soil seed bank, whereas reproductive allocation is a measure of the degree of plant stress, as well as the plant response strategy to stress factors. Primary physiological seed dormancy was used as an indicator of seed bank longevity, whereas seed/seedling vigor as an indicator of initial competiveness of a weed. We hypothesized that drought and shade stress regimes would reduce reproductive output and allocation, as well as result in seed with less intense primary physiological seed dormancy and lower vigor than seed from the non-stressed plants. Likewise, we hypothesized that later maturing seed would be less dormant than early maturing seed. These data will help determine if weeds that mature under resource limiting conditions are likely to produce seed that contribute to the invasiveness and long-term persistence of a species. Such information is necessary to help determine if threshold-based management is appropriate for weeds. 


\section{Results and Discussion}

\subsection{Seed Production and Reproductive Allocation}

In the 2005 wild oat grow out, the drought and shade regimes reduced seed yield and number by nearly $88 \%$ or more compared with the no stress plants (Table 1). Plant biomass and reproductive allocation (RA) was reduced by over $60 \%$ in the drought and shade grown plants compared to the no stress plants. Nearly identical trends were seen in the 2006 wild oat grow out, with the shade regimes reducing seed yields and numbers by $88 \%$ or more, and plant biomass and resource allocation by over $60 \%$.

Table 1. Seed production and reproductive allocation in non-stressed, drought stressed (2005 only) and shade grown wild oat plants in 2005 and 2006. Data for the M73 and SH430 wild oat lines were pooled.

\begin{tabular}{|c|c|c|c|c|c|}
\hline \multirow[b]{2}{*}{$\begin{array}{c}\text { Stress } \\
\text { Environment }\end{array}$} & \multirow[b]{2}{*}{$\begin{array}{l}\text { Maturation } \\
\text { Group }\end{array}$} & \multicolumn{3}{|c|}{ Per Plant } & \multirow[b]{2}{*}{$\mathbf{R A}$} \\
\hline & & Seed Yield & Seed Number & Biomass & \\
\hline 2005 & & g & & g & $\begin{array}{c}\mathrm{g} \text { seed } \\
\mathrm{g} \text { biomass }\end{array}$ \\
\hline \multirow[t]{5}{*}{ No Stress } & 1 & 6.6 & 289 & \multirow{5}{*}{26} & \multirow{5}{*}{1.9} \\
\hline & 2 & 9.5 & 465 & & \\
\hline & 3 & 13.6 & 655 & & \\
\hline & 4 & 17.4 & 840 & & \\
\hline & total & 47 & 2250 & & \\
\hline \multirow[t]{5}{*}{ Drought } & 1 & 0.7 & 38 & \multirow{5}{*}{9} & \multirow{5}{*}{0.6} \\
\hline & 2 & 1.05 & 54 & & \\
\hline & 3 & 1.55 & 81 & & \\
\hline & 4 & 1.9 & 113 & & \\
\hline & total & 5.2 & 286 & & \\
\hline $50 \%$ Shade & Pooled & 1.2 & 65 & 9 & 0.1 \\
\hline $70 \%$ Shade & Pooled & 0.2 & 9 & 4 & $<0.1$ \\
\hline \multicolumn{6}{|l|}{2006} \\
\hline \multirow[t]{3}{*}{ No stress } & Early & 5.6 & 241 & \multirow{3}{*}{15.6} & \multirow{3}{*}{0.8} \\
\hline & Late & 6.6 & 325 & & \\
\hline & total & 12.2 & 566 & & \\
\hline \multirow[t]{3}{*}{$50 \%$ Shade } & Early & 0.7 & 40 & \multirow{3}{*}{5.4} & \multirow{3}{*}{0.2} \\
\hline & Late & 0.6 & 30 & & \\
\hline & total & 1.3 & 70 & & \\
\hline $70 \%$ Shade & Pooled & 0.7 & 72 & 4.6 & 0.1 \\
\hline
\end{tabular}

Numbers in bold represent total values for each stress environment. 


\subsection{Primary Physiological Seed Dormancy}

Analysis of variance indicated that percent germination was significantly $(p<0.01)$ affected by the interaction among stress treatment, maturation group (MG) and after-ripening period for both grow-outs, whereas there were no significant differences among the treatments for the non-viable seed (data not shown). For the 2005 grow-out, germination was near zero percent for the seed subjected to zero to 3 weeks after-ripening in all treatments and MGs (Figure 1). Germination began to approach $20 \%$ by 6 weeks of after-ripening in some of the MGs within the drought treatment, but remained at $5 \%$ or less in the no stress treatments. By 9 weeks of after-ripening, germination ranged from $21 \%$ to $50 \%$ in the seed from the drought stress environment, and from $9 \%$ to $17 \%$ in the no stress treatments. The greatest differences among the treatments were observed with 12 weeks after-ripening, with the highest level of germination (75\%) occurring in the seed from the $\mathrm{MG}_{4}$ in the drought environment, and the lowest $(\approx 20 \%)$ from the $\mathrm{MG}_{1}$ of the no stress environment. Within this after-ripening period, the $\mathrm{MG}_{1}$ of the drought environment and the $\mathrm{MG}_{3}$ of the no stress environment had similar levels of germination which approached 40\%. By 15 weeks of after-ripening, germination was near $80 \%$ for all of the seed from the drought environment, and ranged from $50 \%$ to $71 \%$ in the seed from no stress treatments. Non-viable seed was on average $7.4 \%$ for the entire experiment (data not shown).

Figure 1. Germination of M73 wild oat seed from the 2005 grow-out from plants grown under well-watered (no-stress) and drought conditions after being subjected to varying durations of controlled after-ripening.

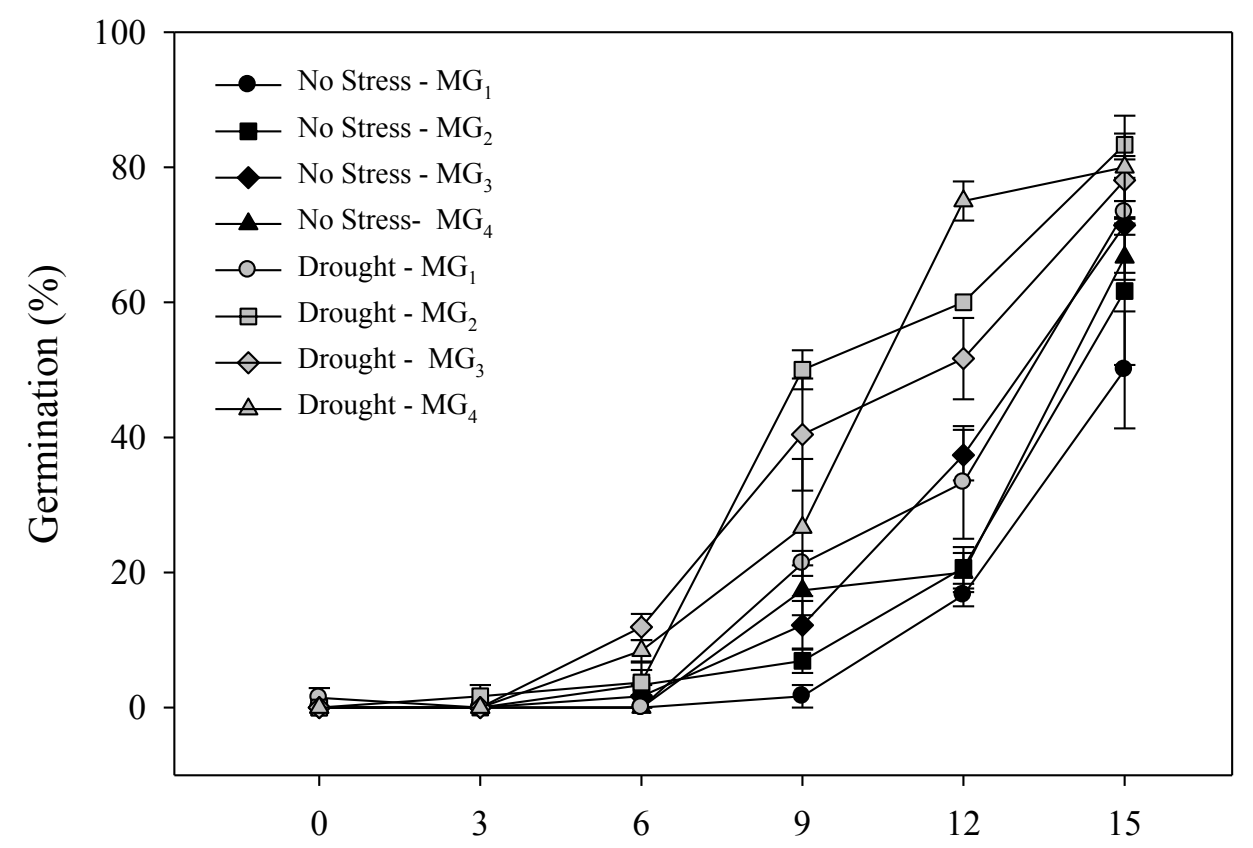

Weeks of After-ripening at $40^{\circ} \mathrm{C} \& 32 \% \mathrm{RH}$

For the 2006 grow-out, germination remained near zero for all treatments with up to 6 weeks of after-ripening (Figure 2). By 12 weeks of after-ripening, germination ranged from $13 \%$ to $24 \%$ in the shade treatments, but near zero in the no-stress treatments. After 18 weeks of after-ripening, germination ranged from $61 \%$ to $81 \%$ in the shade treatments and from $12 \%$ to $30 \%$ in the no stress 
treatments. In this after-ripening period, germination was significantly higher $(p<0.01)$ in the "MG late" compared to the "MG early" in the $50 \%$ shade treatment, but the opposite trend was present between the maturation groups in the seed from the no stress treatments. Non-viable seed was on average $9.0 \%$ for the entire experiment (data not shown).

Figure 2. Germination of M73 wild oat seed from the 2006 grow-out from plants grown under high light (nostress) and shaded conditions after being subjected to varying durations of controlled after-ripening.

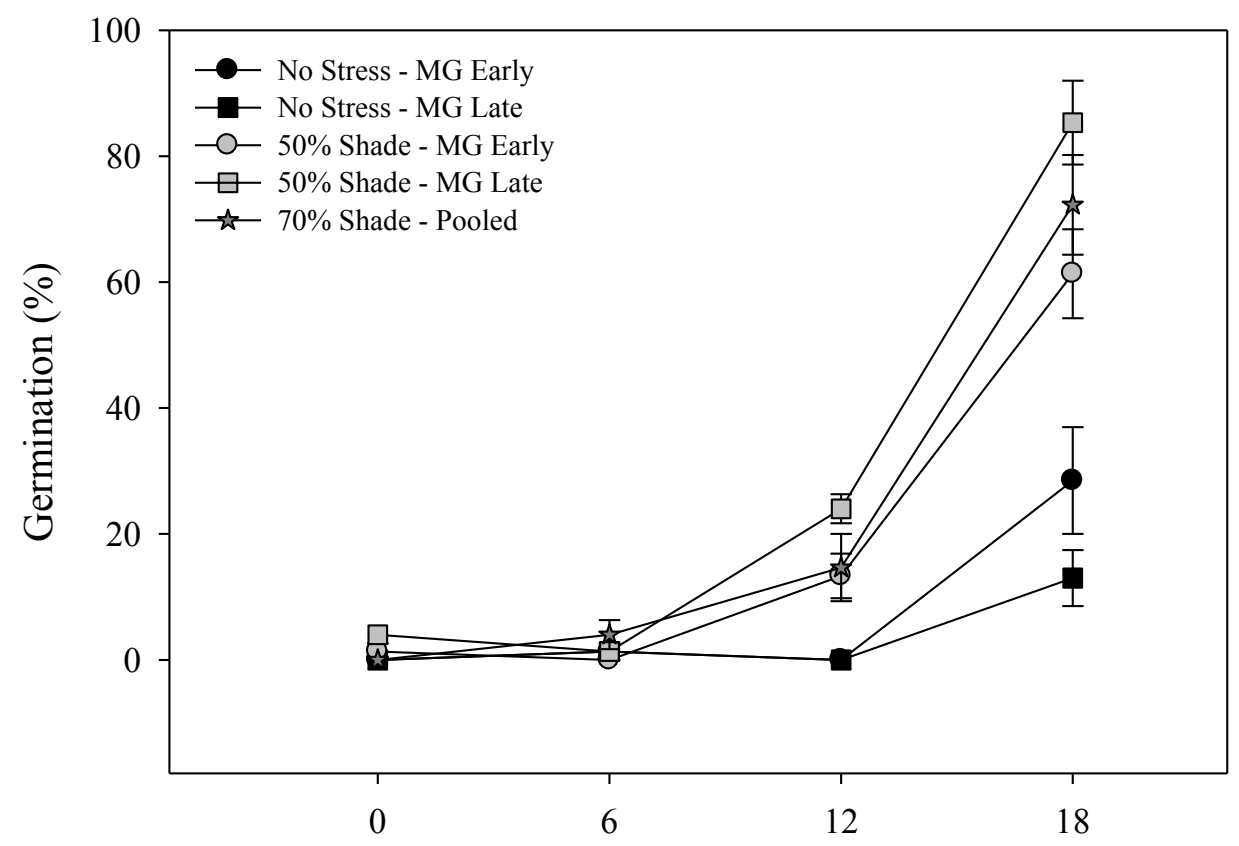

Weeks of After-ripening at $40^{\circ} \mathrm{C} \& 32 \% \mathrm{RH}$

\subsection{Seed and Seedling Vigor}

Analysis of variance indicated that the duration of controlled aging significantly $(p<0.01)$ affected the seed and seedling vigor parameters (Table 2), but there were no discernible differences among the seed maturation environments or MGs. The number of days to seedling emergence did not differ significantly between the zero and 4 weeks aged treatments, averaging approximately 5 days. By 8 weeks of aging however, the days to emergence increased significantly to 7.5 to 8.8 days. The total number of seedlings emerged decreased with each successive aging period, averaging approximately 18 at zero weeks and 7 to 8 days after 8 weeks. Aging duration had no discernible effect on the individual seed biomass, but overall treatment biomass $\#$ seedling emerged $\times$ individual seedling biomass) was highest in the zero and 3 week, and lowest in the 8 week aging regimes. 
Table 2. Mean values for seed vigor variables for the SH430 seed from the 2005 and 2006 grow outs.

\begin{tabular}{ccccc}
\hline $\begin{array}{c}\text { Weeks of } \\
\text { Aging }\end{array}$ & $\begin{array}{c}\text { Days to } \\
\text { Emergence }\end{array}$ & $\begin{array}{c}\text { Total Seedlings } \\
\text { Emerged }\end{array}$ & $\begin{array}{c}\text { Individual Seedling } \\
\text { Biomass }\end{array}$ & $\begin{array}{c}\text { Treatment (trt) } \\
\text { Biomass }\end{array}$ \\
\hline 2005 & $\mathrm{~d} \mathrm{seedling}^{-1}$ & seedlings 14 day $^{-1}$ & mg seedling & ${\text { mg } \operatorname{tr}^{-1}}^{-1}$ \\
0 & $5.1 \mathrm{a}$ & $18.5 \mathrm{a}$ & $17.0 \mathrm{a}$ & $335 \mathrm{a}$ \\
4 & $4.9 \mathrm{a}$ & $15.9 \mathrm{~b}$ & $23.6 \mathrm{a}$ & $370 \mathrm{a}$ \\
8 & $8.8 \mathrm{~b}$ & $8.3 \mathrm{c}$ & $19.9 \mathrm{a}$ & $150 \mathrm{~b}$ \\
pvalue & $<0.0001$ & $<0.0001$ & $n . s$. & $<0.0001$ \\
\hline 2006 & & & & $291 \mathrm{a}$ \\
0 & $5.3 \mathrm{a}$ & $17.8 \mathrm{a}$ & $15.6 \mathrm{a}$ & $284 \mathrm{a}$ \\
4 & $5.1 \mathrm{a}$ & $13.7 \mathrm{~b}$ & $20.8 \mathrm{a}$ & $121 \mathrm{~b}$ \\
8 & $7.5 \mathrm{~b}$ & $6.6 \mathrm{c}$ & $18.2 \mathrm{a}$ & $<0.0001$ \\
\hline pvalue & $<0.0001$ & $<0.0001$ & $n . s$. & \\
\hline
\end{tabular}

Values within each year by aging duration were pooled among maturation treatments; Means followed by the same letters are not significantly different according to a Least Squared means separation test $(p<0.05)$.

\subsection{Discussion}

The drought and shade regimes imposed in this experiment clearly had an effect on wild oat plant growth and reproduction, substantially reducing plant biomass, reproductive output and reproductive allocation relative to the wild oat plants grown under resource rich conditions (Table 1). As previously reported in [7] these stress environments also reduced the mass of the caryopsis and hull by up to $21 \%$ and $43 \%$, respectively, and the total phenolic acid content of the hull by up to $45 \%$. Within a species, environmental regulation of seed size is common and has been suggested to have important implications for seed vigor and establishment, with seedling survival often directly correlated to seed mass (reviewed by [8]). In our experiment with wild oat, we did not see any obvious differences in seed or seedling vigor in seed from the maturation environments tested (Table 2). This may be due to the controlled laboratory aging protocol used in this study did not adequately mimic in situ seed aging. Seed bank longevity of wild oat has been estimated to be as long as 10 years [9], and large differences in emergence phenology and seed bank persistence among seed from different maturation environments has been observed in in situ studies [6]. We used the controlled aging protocol to enable us to make a more rapid evaluation of seed vigor than could be accomplished with an in situ approach. More work may be needed to refine laboratory aging protocols to better mimic the environmental, chemical and biological stressor that result in the gradual loss of seed vigor and viability in soil seed banks [4]. Preliminary evaluation by our collaborators did suggest the wild oat seed from the stressed environments were more prone to infection by various strains of Fusarium when inoculated by methods as outlined in [10] (A.C. Kennedy, personal communication). The lower concentration of phenolic acids in the stress-grown seed [7] may help explain these trends [4].

The drought and shade regimes, as well as maturation timing, significantly affected the degree of primary physiological dormancy of the wild oat seed, as indicated by the germination trends throughout the controlled after ripening periods (Figures 1 and 2). The germination differential was as much as $\sim 60 \%$ between the drought-stressed seed $\left(\mathrm{MG}_{4}\right)$ and the non-stressed seed $\left(\mathrm{MG}_{1}\right)$ after 
12 weeks of accelerated after-ripening (Figure 1). These results are consistent with the findings reported in [5] where wild oat seed from drought-stressed plants were considerably less dormant than seed from non-stressed plants, which can result in large differences in emergence phenology and seed bank persistence in the field [6]. Similarly in our experiment, shaded plants produced seed that were considerably less dormant than the seed from plants grown under normal light conditions, with a germination differential of over $70 \%$ between the seed from the shade (MG late) and the "no-stress" (MG late) after 18 weeks of accelerated after-ripening (Figure 2). Although the plants from the 70\% shade treatment tended to produce fewer seed per plant than the plants from the $50 \%$ shade treatment (Tables 1 and 2), there was no evidence that this additional level of light limitation affected seed dormancy to a greater degree than the 50\% shade treatment (Figure 2). For other weed species, the reported effects of shade on physiological seed dormancy have been mixed; increasing dormancy in eastern black nightshade (Solanum ptycanthum Dun.) [11] and buckhorn plantain (Plantaga lanceolata L.) [12]; reducing dormancy in velvetleaf (Abutilon theophrastii L.) [13]; or having no effect on dormancy in johnsongrass (Sorghum halepense L.), and jimsonweed (Datura stramonium L.) [14]. We acknowledge that greenhouse shade cloth used in our experiment and others cited in this report does not differentially absorb red light compared to far-red light as would be the case with vegetative shade [15]. However, the observed plant growth characteristics of our shade-grown plants were similar to those we would expect from plants grown in vegetative shade, including increased stem elongation and delayed flowering. Since our experiment was conducted in pots, it would not have been meaningful to measure the root to shoot ratio of our plants, which tends to decrease under light-limiting conditions. Since the grow outs for the seed in the drought and shade regimes used in this experiment were conducted separately in different locations, we cannot make definitive conclusions about the relative effects of drought stress and light limitation during seed development and maturation on primary seed dormancy. In addition to the effects of drought and shade stress on primary dormancy in wild oat, it appears that seed maturation timing within a growth regime also can influence seed dormancy. Within the drought stress treatments, there was $41 \%$ higher germination in the $\mathrm{MG}_{1}$ compared to the $\mathrm{MG}_{4}$ at the 12 week after-ripening periods (Figure 1). Similar, although less pronounced, trends among the maturation groups were evident in the no stress and shade treatment at some of the after-ripening periods (Figure 2). These data suggest that variability in primary dormancy of wild oat in the soil weed seed bank is likely a function of plant genetics [16], plant resource availability, and seed maturation timing.

From this experiment, we cannot precisely predict the impact that water and light limitation during wild oat seed development would have on the emergence phenology of these seed in subsequent years. In our experiment, accelerated after-ripening periods of 15 weeks or more tended to alleviate much of the dormancy in the stress-grown seed, but considerable dormancy remained in the seed from the non-stressed environments (Figures 1 and 2). Based on temperature alone, these periods would translate to a heat unit accumulation in excess of 4000 degree days, assuming a base temperature of $0{ }^{\circ} \mathrm{C}$. Seed grown under field conditions, however, would be subjected to both variable temperature and soil water content, which tend to interactively regulate the alleviation of primary dormancy [17-19]. Likewise, we utilized the M73 wild oat line in order to minimize genetic variability in our experiments. The M73 line, however, tends to be more dormant than many wild-type seed populations [20]. Finally, we imposed single stress factors in our experiment in order better understand the mechanistic 
contribution of each stress factors to seed dormancy, among other seed traits not discussed in this report. Wild oat under field conditions, however, would likely experience multiple stress factors due to competition with the crop that could occur simultaneously. To more conclusively understand the implications of plant stress during seed development and maturation on seed dormancy in wild oat, it would be important to conduct similar experiments under field conditions. In studies with other weed species, field-based competition did produce measureable effects on seed persistence traits. For example, Nurse and DiTommaso [21] found that velvetleaf grown in competition with corn produced seed that were up to $30 \%$ less dormant and with lower seed and seed coat weights than plants grown without competition. Similarly, it has been shown there can be significant variability in velvetleaf seed persistence depending on the maternal source of the seed [22]. In addition to variability in soil and climatic condition, factors such as photoperiod and nutrient availability can influence the level of primary dormancy in some weed species [4].

\section{Experimental Section}

\subsection{Plant Materials and Stress Environments}

The wild oat line "M73" was used in dormancy components of this study due to its pronounced primary seed dormancy, whereas the "SH430" line was used in the seed/seeding vigor component of the study due to is lack of primary physiological seed dormancy [20]. The seed initially used in this experiment were obtained from a field-based grow-out conducted at the Palouse Conservation Field Station and Agricultural Research Service (Pullman, WA, USA) in the summer of 2002. Plants were grown under full-sun and well-watered conditions and the seed were stored at $-20{ }^{\circ} \mathrm{C}$ until the initiation of this experiment. Prior to starting the greenhouse grow-outs for this experiment, the dormancy in these seed was alleviated by an 8 week accelerated after-ripening protocol described in the "Seed Dormancy and Viability" section below. The first grow-out for this experiment was conducted from July to November 2005 in a greenhouse at the plant growth facilities located at Washington State University (WSU), Pullman WA, and consisted of: (1) no stress; (2) drought stress; (3) $50 \%$ shade; and (4) 70\% shade treatment regimes. Since we anticipated a large difference in the total number of seed per plant produced among the stress treatments, a different number of pots (1 plant per pot) were used for each regime. There were 31 pots for the no stress; 168 pots for the "drought stress"; 50 pots for the " $50 \%$ shade"; and 140 pots for " $70 \%$ shade". Pots were arranged in single stress treatments clusters, separated by polyester row cover, to prevent future cross pollination [23]. Pots measuring $10 \times 10 \times 35 \mathrm{~cm}$ (Stuewe and Sons, Inc., Tangent, OR, USA) were filled $5 \mathrm{~cm}$ from the top with Sun-Gro Sunshine ${ }^{\circledR}$ Mix \#1 (SunGro ${ }^{\circledR}$ Horticulture, Vancouver, British Columbia, CA, USA). The mix for each pot contained $\sim 15 \mathrm{~g}$ of Osmocote $^{\circledR}$ (19:6:12) slow release fertilizer which was supplemented with biweekly additions of $\sim 100 \mathrm{~mL}$ Miracle-Gro ${ }^{\circledR}$ soluble fertilizer solution $(1 \% \mathrm{w} / \mathrm{v} ; 18: 18: 21+$ micronutrients) per pot. Multiple seed (3-4) were planted per pot and thinned to 1 per pot after 2 weeks. Stress regimes were not imposed until plants reached the 5 tiller stage.

Drought conditions were simulated by imposing a cyclical watering regime during which the pots were watered to container capacity based on gravimetric water content, allowed to dry to $\sim 30 \%$ 
capacity for 2 to 3 days and then re-watered to container capacity, for total of six cycles (Figure 3). This protocol was based on preliminary experiments conducted to ascertain the degree of drought stress that could be imposed without causing plant death. The gravimetric water content was determined by weighing 3 indicator pots for each line, but did not factor out the wild oat biomass accumulation in the calculation, which was determined to be small compared to the weight of the potting mix and water. The no stress and shade treatment pots were maintained at a water content of $80 \%$ of container capacity or higher throughout the grow-out periods. The shade treatments were imposed by using black shade cloth (50\% and 70\%; Gempler, Madison, WI, USA) that was supported on a PVC pipe framework completely surrounding shade treatment clusters. This shade cloth had a negligible effect $(<2 \%)$ on the red to far-red light ratio of the light reaching the plants $(660 / 730$ Sensor, Skye Instruments, Wales, UK), but is reported to reduce ultraviolet light levels, according to the manufacturer. Aphid pests (species unidentified) were controlled as needed using registered insecticides applied at labeled rates. Greenhouse temperatures ranged from 25 to $35^{\circ} \mathrm{C}$ for the duration of the grow-out period, and natural lighting was supplemented with six $400 \mathrm{~W}$ high pressure sodium lamps per $1.2 \times 2.4 \mathrm{~m}$ greenhouse bench, set to a $16 \mathrm{~h}$ photoperiod.

Figure 3. Gravimetric soil water content throughout the life cycle of the drought-grown wild oat plants. Error bars represent one standard error of the mean.

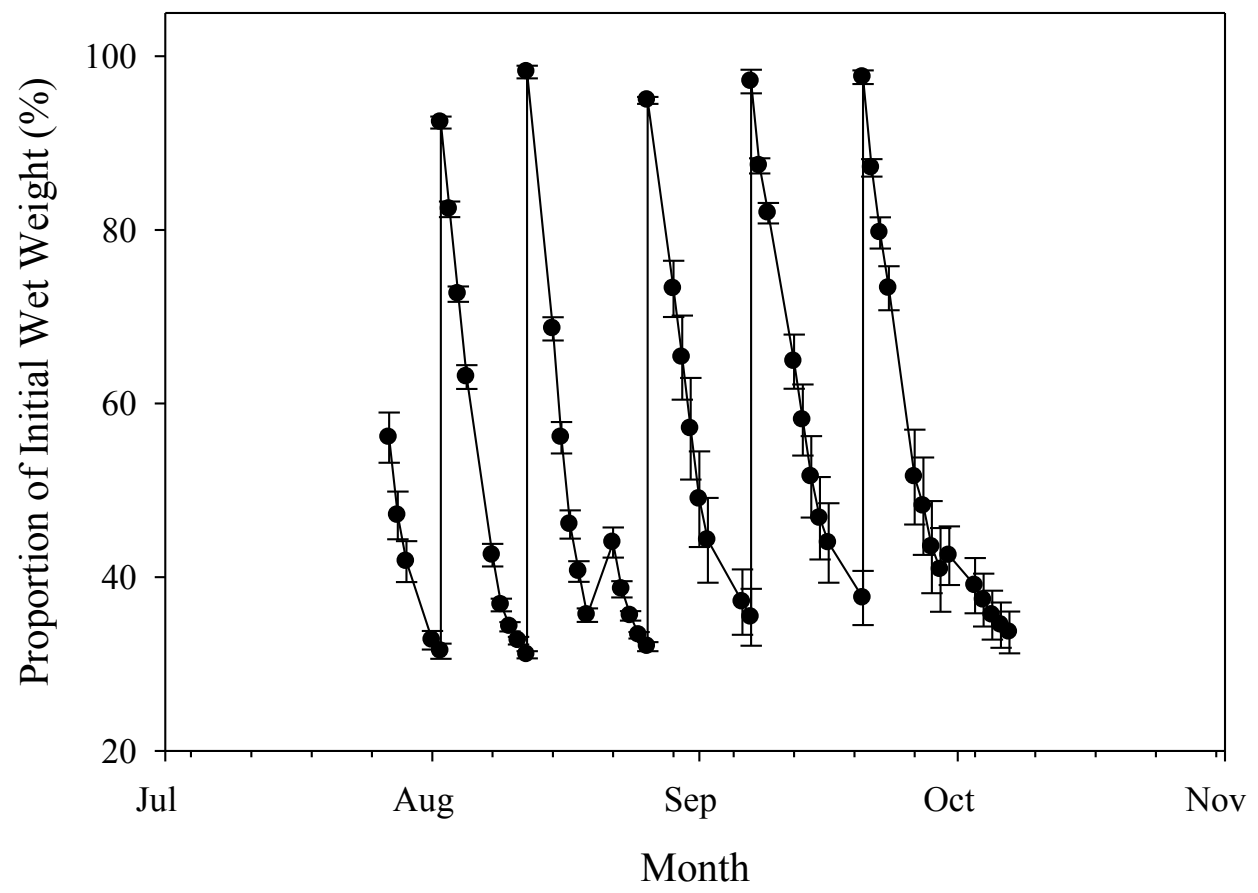

Seed were harvested daily for each stress treatment cluster beginning in late August and ending in early November. Seed were removed from the panicle by gently shaking the panicle and catching seed in a small wash basin. Weekly seed pools were kept separate and stored at $-20{ }^{\circ} \mathrm{C}$. After all the seed had been harvested, plants were dried in the greenhouse for 3 weeks in the absence of any climate control, with day temperatures typically exceeding $35{ }^{\circ} \mathrm{C}$. Biomass for the entire stress treatment cluster was measured after this drying period. Chaff was cleaned from the weekly seed harvest pools by passing the seed through a home-made forced air seed cleaning device, which removed the seed that 
did not contain a caryopsis, as well as the chaff and other plant debris. The weekly seed harvests from the no stress and "drought stress" treatment clusters were pooled into four maturation groups $\left(\mathrm{MG}_{1-4}\right)$, based on total seed weight; attempting to achieve four groups of near equal mass. The shade treatments did not produce a sufficient number of seed to divide the seed pools into maturation groups, or sufficient seed to complete the desired suite of experiments. As such, an additional grow-out for the shade and the no stress treatments was repeated in 2006 (July-November) at the greenhouses associated with the Department of Crop and Soil Sciences at Penn State (University Park, PA, USA) under similar growth conditions and methodologies as used in the WSU grow-out. For the 2006 grow-out, the weekly seed harvests were grouped into two pools: early or late maturing, with near equal total seed masses between the pools. Seed mass for both grow-outs was determined by taking 3 allocations of 50 seed from each maturation group from the stress treatment clusters. Seeds were lyophilized to determine the dry weight.

\subsection{Seed Dormancy and Viability}

The level of primary seed dormancy in the M73 wild oat seed from the stress treatments was evaluated by controlled after-ripening methods similar to those outlined in [17]. From each MG seed lot in the no stress and "drought stress" treatments for the 2005 grow-out and the no stress and shade treatments for the 2006 grow-out, three pools or replicates of 250 seed each were suspended in a sealed desiccator jar above a saturated $\mathrm{MgCl}_{2} \cdot 6 \mathrm{H}_{2} \mathrm{O}$ solution, which yields a relative humidity of $32 \%$ at $40{ }^{\circ} \mathrm{C}$ [24] and a seed moisture content of $\sim 10 \%$. There was insufficient seed to evaluate seed dormancy in the shade treatments from the 2005 grow-out. The desiccator jar was placed in a water bath set to $40{ }^{\circ} \mathrm{C}$ with a fabricated insulated cover that left only the top portion of the desiccator exposed. For each after-ripening period, 25 seed were removed from each replicate seed pool and plated on a $9 \times 9 \mathrm{~cm}$ square petri plate containing $1 \%$ non-nutrient agar. Petri plates were sealed with Parafilm $^{\circledR}$ (Pechiney Plastic Packaging, Menasha, WI, USA) to prevent desiccation of the agar. Petri plates were placed in a controlled environment chamber (Model SG30SC, Hoffmann Manufacturing Inc., Jefferson, OR, USA) set to a day temperature of $25{ }^{\circ} \mathrm{C}$ and a night temperature of $15{ }^{\circ} \mathrm{C}$ for $12 \mathrm{~h}$ each. The $12 \mathrm{~h}$ photoperiod corresponded with the day temperature, with light levels of 40 to $50 \mu \mathrm{mol} \mathrm{m} \mathrm{m}^{-2} \mathrm{~s}^{-1}$ (PAR) delivered from six fluorescent units within the chamber. Germination was tallied over a 14 day period and was based on the emergence of a root radical from the seed. Seed that did not germinate were tested for viability with a $1 \%$ tetrazolium (2,3,5-triphenyl- $H$-tetrazolium chloride) solution [25]. The after-ripening duration consisted of 3 week intervals up to 15 weeks for the 2005 grow-out, and 6 week intervals up to 18 weeks for the 2006 grow-out. Seed within each replicate seed pool not subjected to any laboratory-induced after-ripening served as the control.

\subsection{Seed Vigor Evaluation}

To simulate the natural decrease in seed vigor that occurs in the soil seedbank over time, controlled seed aging was achieved by placing the seed in glass mason jars suspended over a saturated $\mathrm{NaNO}_{3}$ solution. The jars were sealed and then placed in a water bath at $35{ }^{\circ} \mathrm{C}$, creating a $74 \%$ relative humidity environment [24] and an approximate seed water content of $\sim 15 \%$. Seed were exposed to this treatment for 0,4 , and 8 weeks. The jar configuration for each maturation environment was identical to 
that outlined for the controlled after ripening experiment in section 3.2 of this paper. After aging period, 20 seeds from each maturation environment replicate pool were removed from the jars and planted in plug flats $\left(12.5 \mathrm{~cm}^{3}\right.$ well, ITML Horticultural Products Inc, Ontario, Canada) containing Sunshine Potting Mix \#2 containing no added nutrients. The flats were placed in a Conviron growth chamber set at $200 \mu \mathrm{mol} \times \mathrm{m}^{-2} \times \mathrm{s}^{-1}$ and $22{ }^{\circ} \mathrm{C}$. Coleoptile emergence for each seed was tracked daily for two weeks. After two weeks of growth after coleoptile emergence, each seedling was cut at soil level and dried in an oven at $80{ }^{\circ} \mathrm{C}$ for at least two days, after which it was weighed to determine biomass. The measurements included days to emergence, number of seedling emerged, the percentage of total seedlings emerged and biomass per seedling.

\subsection{Statistical Analysis}

The percent germination and non-viable seed for the seed dormancy experiment and percentage emergence in the seed vigor experiment were calculated based on the total number of seed in each Petri plate. Analysis of variance was conducted for percent germination and percent non-viable seed using a general linear model (GLM) procedure in SAS Version 9.1 (SAS Institute, Cary, NC, USA) using stress environment, MG (seed dormancy experiment only) and after-ripening/aging period as categorical independent variables. For the seed and seedling mass data, analysis of variance was conducted using a GLM procedure using stress environment and MG as a categorical independent variables. Data for the two grow-outs were analyzed separately. For the percent germination and emergence analysis, the data were transformed using an arcsine square root transformation to correct for heterogeneity of variance. The data for the percent non-viable seed and seed mass did not require this transformation due to acceptable homogeneity of variance in these data.

\section{Conclusions}

In conclusion, our data support the hypothesis that seed dormancy within a species is a highly plastic trait that can be strongly influenced by the growth conditions of the mother plant. The principles of threshold-based weed management indicate that low levels of weed competition often do not cause serious crop yield losses and may not merit the implementation of active weed control strategies [26]. Although the concerns that sub-threshold weed populations may contribute to the long-term weed seed bank are duly noted [3], there is a growing body of data suggesting that primary seed dormancy and secondary metabolites in seed as seed bank persistence mechanisms may be greatly curtailed in sub-threshold weed populations maturing under resource-limiting conditions. As such, precocious germination, which can be easily managed with broad-spectrum herbicides or mechanical means, or rapid seed death is likely to occur in seed bank additions from sub-threshold stress grown weeds. Conversely, weeds that are not subject to intense competition from the crop are likely have greater and persistent seed bank additions, which will be the source of future weed infestations. Understanding the plasticity of seed traits, such as seed dormancy, vigor and chemistry, in relation to maturation environment will help better delineate the implementation criteria for reduced input weed management strategies that prevent the proliferation of future weed communities. Less reliance on post emergence herbicide programs can be an important part of a management plan to slow the development of herbicide resistant weed communities. 


\section{Acknowledgments}

Funding for the research was provided in part by the USDA NRI Biology of Weedy and Invasive Species Program (Award No. 2005-35320-15375), The Pennsylvania State University, and Washington State University. Special thanks to Wendy McCoy, Lidewij Keser, Jarrus Rossi, and Lindsay Koller, for their technical support on these aspect of the project.

\section{Conflict of Interest}

The authors declare no conflict of interest.

\section{References}

1. Gilliom, R.J. Pesticides in U.S. streams and groundwater. Environ. Sci. Technol. 2007, 41, 3408-3413.

2. Jasieniuk, M.J.; Brule-Babel, A.L.; Morrison, I.N. The evolution and genetics of herbicide resistance in weeds. Weed Sci. 1996, 44, 176-193.

3. Norris, R.F. Ecological Implications of Using Thresholds for Weed Management. In Expanding the Context of Weed Management; Buhler, D.D., Ed.; Hawthorn Press: New York, NY, USA, 1999; pp. 31-58.

4. Gallagher, R.S.; Fuerst, E.P. The Ecophysiology Basis of Weed Seed Longevity in the Soil. In Seed Science and Technology; Basra, A.S., Ed.; Haworth Food Products Press: Binghamton, NY, USA, 2006; pp. 521-557.

5. Sawhney, R.; Naylor, J.M. Dormancy studies in seed of Avena fatua 13: Influence of drought stress during seed development on the duration of seed dormancy. Can. J. Bot. 1982, 60, 1016-1020.

6. Peters, N.C.B. Production and dormancy of wild oat (Avena fatua) seed from plants grown under water stress. Ann. Appl. Biol. 1982, 100, 189-196.

7. Gallagher, R.S.; Granger, K.L.; Keser, L.H.; Rossi, J.; Pittmann, D.; Rowland, S.; Burnham, M.; Fuerst, E.P. Shade and drought stress-induced changes in phenolic content of wild oat (Avena fatua L.) seed. J. Stress Physiol. 2010, 6, 90-107.

8. Leishman, M.R.; Wright, I.J.; Moles, A.T.; Westoby, M. The Evolutionary Ecology of Seed Size. In Seed: The Ecology of Regeneration in Plant Communities, 2nd ed.; CABI Publishing: Wallingford, UK, 2000; pp. 31-58, 410.

9. Simpson, G.M. Seed Dormancy in Grasses; Cambridge University Press: Cambridge, UK, 1990; p. 308.

10. De Luna, L.Z.; Kennedy, A.C.; Hansen, J.C.; Paulitz, T.C.; Gallagher, R.S.; Fuerst, E.P. Mycobiota on wild oat (Avena fatua L.) seed and their caryopsis decay potential. Plant Health Progr. 2011, doi:10.1094/PHP-2011-0210-01-RS.

11. Stoller, E.W.; Myers, R.A. Effects of shading and soybean Glycine max (L.) interference on Solanum ptycanthum (Dun.) (eastern black nightshade) growth and development. Weed Res. 1989, 29, 307-316. 
12. Van Hinsberg, A. Maternal and ambient environmental effects of light on germination in Plantaga lanceolata: Correlated responses to selection on leaf length. Funct. Ecol. 1998, 12, 825-833.

13. Bello, I.A.; Owen, M.; Hatterman-Valenti, H.M. Effect of shade on velvetleaf (Abutilon theophrasti) growth, seed production, and dormancy. Weed Technol. 1995, 9, 452-455.

14. Benvenuti, S.; Macchia, M.; Stefani, A. Effects of shade on reproduction and some morphological characteristics of Abutilon theophrasti Medicus, Datura stramonium L. and Sorghum halepense L. Weed Res. 1994, 34, 283-288.

15. Turnbull, C.G.N. Shoot architecture II: Control of Branching. In Plant Architechture and Its Manipulation: Annual Plant Reviews 17; CRC Press: Boca Raton, FL, USA, 2005; pp. 92-120.

16. Foley, M.E.; Fennimore, S.A. Genetic basis for seed dormancy. Seed Sci. Res. 1998, 8, 173-182.

17. Foley, M.E. Temperature and water status affecting afterripening in wild oat (Avena fatua). Weed Sci. 1994, 42, 200-204.

18. Steadman, K.J.; Crawford, A.D.; Gallagher, R.S. Dormancy release in Lolium rigidum seed is a function of thermal after-ripening time and seed water content. Funct. Plant Biol. 2003, 30, 345-352.

19. Gallagher, R.S.; Crawford, A.D.; Steadman, K.J. Alleviation of dormancy in annual ryegrass (Lolium rigidum) seed by hydration and after-ripening. Weed Sci. 2004, 52, 968-975.

20. Adkins, S.W.; Loewen, M.; Symons, S. Variation within pure lines of wild oats (Avena fatua) in relation to degree of primary dormancy. Weed Sci. 1986, 34, 859-864.

21. Nurse, R.E.; DiTommaso, A. Corn competition alters the germinability of velvetleaf (Abutilon theophrasti) seed. Weed Sci. 2005, 53, 479-488.

22. Schutte, B.J.; Davis, A.S.; Renner, K.A.; Cardina, J. Maternal and burial environment effects on seed mortality of velvetleaf (Abutilon theophrasti) and Giant Foxtail (Setaria faberi). Weed Sci. 2008, 56, 834-840.

23. Imam, A.G.; Allard, R.W. Population studies in predominantly self-pollinated species IV: Genetic variability between and within natural populations of wild oats from different habitats in California. Genetics 1965, 51, 49-62.

24. Dhingra, O.D.; Sinclair, J.B. Basic Plant Pathology Methods, 2nd ed.; CRC Press: Boca Raton, FL, USA, 1995; p. 434.

25. AOSA (Association of Official Seed Analysis). Tetrazolium Testing Handbook: Contribution No. 29 to the Handbook on Seed Testing; Peters, J., Ed.; The Association of Official Seed Analysts: Lincoln, NE, USA, 2000.

26. Coble, H.D.; Mortensen, D.A. The threshold concept and its application to weed science. Weed Technol. 1992, 6, 191-195.

(C) 2013 by the authors; licensee MDPI, Basel, Switzerland. This article is an open access article distributed under the terms and conditions of the Creative Commons Attribution license (http://creativecommons.org/licenses/by/3.0/). 